\title{
Do social relationships mediate or moderate social inequalities in health? A systematic review protocol
}

Nadia Khaliq ( $\square$ nadia.khaliq.16@ucl.ac.uk)

UCL: University College London https://orcid.org/0000-0002-7538-7110

Anne McMunn

UCL: University College London

Carolina Machuca-Vargas

King's College London

Anja Heilmann

UCL: University College London

\section{Protocol}

Keywords: Public Health, Social determinants of health, Epidemiology, Social relationships

Posted Date: October 13th, 2020

DOl: https://doi.org/10.21203/rs.3.rs-89905/v1

License: (c) (i) This work is licensed under a Creative Commons Attribution 4.0 International License. Read Full License 


\section{Abstract}

Introduction

Explanations for health inequalities include material, behavioural and psychosocial pathways. Social relationships are an important determinant of health, and research has consistently found that a lack of support networks may diminish favourable health outcomes. There is some evidence that social network structures, partly shaped by socioeconomic factors, contribute to health inequalities. This protocol will summarise the systematic review process.

Methods and analyses

The Systematic review will be reported according to the Preferred Reporting Items for Systematic Reviews and Meta-Analyses guidelines. An electronic database search of MEDLINE, Embase Classic + Embase, and PsychINFO using the OvidSP platform will be undertaken. Articles published in the English language that have quantitatively assessed the role of social relationships in mediating or moderating health inequalities will be included and any health outcome (mental/physical) will be considered. The database search will be supplemented by reference list screening of all relevant full-text articles identified through the search. Two independent reviewers will be responsible for screening of articles, data extraction and assessment of bias. Studies will be risk assessed for bias using a modified version of the NewcastleOttawa Quality Assessment Scale. It is anticipated that the eligible studies will be highly variable; therefore, a meta-analysis will only be considered if the available data of the selected studies are similar. If the studies are too heterogeneous, a narrative synthesis of the extracted data will be presented.

\section{Conclusion}

The results of the systematic review will examine the link between social relationships and health inequalities. The findings of the review will identify gaps in knowledge where further research is needed.

Systematic review registration

PROSPERO CRD42020181706

\section{Background}

\section{Social relationships and health}

Social inequalities in health are universally recognised as a significant public health problem (1). The WHO conceptual framework for action on the social determinants of health postulates that structural determinants, including the socioeconomic position of individuals, shape health outcomes through the material, psychosocial and biological factors (intermediate determinants). According to this framework, psychosocial factors include social relationships, with social cohesion and social capital seen as cutting across the structural and intermediary determinants to influence health inequalities (2) 
In the UK, tackling health inequalities is an important national strategy. The publication of the White Paper, 'Healthy Lives, Healthy People'(3) in response to the Marmot Review (4) led to fundamental legislative change in the government's approach to tackling the broader structural determinants of health. This change in legislation led to the Health and Social Care Act (5), mandating health bodies, including Public Health England, to reduce health inequalities (6). However, whilst material and behavioural pathways between socioeconomic position and health have been studied extensively, few studies have explored the contribution of social relationships to social gradients in health.

The importance of social relationships for health and well-being is well established $(7,8)$. Social relationships broadly refer to the degree of connection and ties individuals have with others (8) and include a range of different concepts such as social support, social networks, and social cohesion. A meta-analysis by Holt-Lunstad et al. (7) concluded that the influence of social relationships on mortality risk is comparable in magnitude to the impact of other well-known risk factors such as obesity and health-compromising behaviours. More unsatisfactory social relationships have also been linked to an increased incidence of coronary heart disease and stroke (9), higher levels of inflammatory markers (10), poorer self-rated oral health and oral-health related quality of life (11), depression (12), cognitive decline (13-15) and altered host susceptibility to disease due to the effect on immune function and neuroendocrine responses (16-18).

Evidence of the mediating and moderating role of social relationships in explaining health inequalities

Despite the vast literature on the importance of social relationships for health, evidence regarding the role of social relationships in explaining health inequalities is limited, and results have been contradictory (1920).

Evidence suggests potential interactions between markers of socioeconomic position and social relationships and their collective impact on social inequalities in health. Several previous studies assessed whether social relationships can weaken or strengthen the associations between socioeconomic position and health, but results have been highly variable (21-26).

To date, there has been no comprehensive review of the literature on the role of social relationships in mediating or moderating health inequalities. Existing reviews have focused on the role of social capital (27-29). Carlson and Chamberlain (27) and Vyncke et al. (29) reviewed the role of neighbourhood-level social capital in explaining social inequalities in health. Vyncke et al. (29) limited the population group to children and adolescents, and Carlson and Chamberlain (27) focused on civic trust alone. Similarly, Uphoff et al. (28) only examined whether social capital moderates socioeconomic inequalities in health.

\section{Research aim}

This systematic review aims to assess and synthesise the evidence on the role of social relationships in the association between socioeconomic position and health. The main objectives are: 
1. To assess the evidence for the mediating role of social relationships in the association between socioeconomic position and health;

2. To assess the evidence for the moderating role of social relationships in the association between socioeconomic position and health.

\section{Methodology}

\section{Protocol and registration}

This protocol is registered with PROSPERO (CRD42020181706) and has been reported according to the Preferred Reporting Items for Systematic Reviews and Meta-Analyses Protocols (30) (see Additional File 1).

\section{Study eligibility criteria}

\section{Participants}

Individuals from any population group and of all ages will be included - there will be no age restrictions or cut-offs for including or excluding studies in this review. Individuals with any physical or mental health condition will also be included.

\section{Study design}

The review will include any interventional or observational study that assesses the role of social relationships in mediating or moderating health inequalities. Studies need to have quantified the contribution of social relationships to the association between socioeconomic position and health (for mediation) or presented interaction effects or stratified results for different levels of social relationships (for moderation).

Studies will be included if they are original studies published in peer-reviewed journals that examine the mediating or moderating role of at least one indicator of social relationships in the association between socioeconomic position and health.

Studies will be excluded if they are qualitative studies, review articles, grey literature, opinion pieces or theoretical articles. Additionally, studies will also be excluded if they only adjusted for socioeconomic position rather than test for the mediating or moderating role of social relationships in explaining health inequalities.

\section{Outcomes}

This review will include studies that cover a wide range of subjective or objective measures of mental and physical health when assessing health inequalities. Outcome measures will include, but will not be 
limited to, mortality, coronary heart disease, stroke, immune response, self-rated health, health-related quality of life, depression, cognitive decline and measures of oral health. The inclusion and exclusion criteria will not be based on the type of outcome measure used; however, these will be recorded when extracting the data.

\section{Exposures}

The exposures of interest are socioeconomic position and social relationships. The review will focus on the combined impact of these exposures, i.e. studies will only be included if they explored whether social relationships mediated or moderated the association between socioeconomic position and health.

Socioeconomic position summarises an individual's position within the social hierarchy and refers to their material and social resources (2). Studies will not be excluded based on the type of the socioeconomic variable used. Any measure of socioeconomic position will be included such as occupational status, educational level, income or household wealth; as well as proxy measures such as housing tenure or car ownership, where the indicators mentioned above are not available.

There is no universally agreed-upon definition of what constitutes social relationships. Therefore, the search strategy will intentionally include terms that refer to the degree of connection individuals have with others, such as social connectedness, social support, social networks, engagement, sociability, social attachments, social capital and social integration.

Studies may differentiate between structural and functional aspects of social relationships. Structural elements refer to the quantitative part of social relationships and include measures such as marital status or social network size (31). Functional aspects refer to the qualitative part of social relationships and include measures such as loneliness and isolation (31).

Studies will not be excluded based on the type, reliability and validity of the social relationship measure used.

Only articles published in the English Language will be retrieved. No date restrictions will be applied to the search strategy, and databases will be searched from the earliest date of entry until $1^{\text {st }}$ October 2020.

\section{Search strategy}

An electronic search will be carried out to identify all relevant studies from the following databases: MEDLINE, Embase Classic + Embase and PsycINFO.

Search terms include terms relating to social relationships, socioeconomic position and health inequalities. Specific health outcomes will not be included in the search strategy, as any health outcome will be considered (see Additional File 2 for search terms table). 
Various tools such as Boolean operators, truncation and proximity indicators will be used to ensure a comprehensive search that identifies all relevant articles (see Additional File 3). Reference lists of all relevant full-text papers will be screened to retrieve additional articles not identified through the database search. The study selection process will be summarised in a PRISMA flowchart (32).

\section{Study selection}

All relevant articles retrieved from the database searches will be stored in the reference manager, Mendeley (33). Articles will be exported to Rayyan QCRI (34) after removal of duplicates. Two reviewers (NK and CMV) will independently screen titles and abstracts for eligibility. The UCL findit@UCL linking service will be used to retrieve full texts of relevant articles. Any disagreements regarding the inclusion of papers will be resolved through discussions. Any remaining unresolved disputes following this process will go through a tie-break decision from an external reviewer not involved in the review process.

\section{Data extraction}

A data extraction form will be developed and piloted using a sample of eligible studies. The piloting process will ensure reliability in the interpretation and use of the inclusion criteria. Upon finalisation of the data extraction form, the reviewers (NK and CMV) will extract the data and reasons for exclusion will be listed. Any discrepancies will be resolved through consensus meetings.

The reviewers will extract data regarding key elements of each study, including:

- Citation details such as first author and year of publication;

- Study population including country or region, sample size and demographic indicators such as age and sex;

- Study design, e.g. cross-sectional, longitudinal;

- The follow-up period for longitudinal designs;

- Whether the study examined mediation or moderation;

- Exposure indicators and health outcomes assessed;

- Statistical methods implemented and main results, e.g. ORs, relative risks.

\section{Synthesis}

It is anticipated that the eligible studies to be included in the review will be highly variable with regards to populations, settings, study design and outcomes. A meta-analysis will be considered if the available data of the selected studies are similar with regards to populations, settings, study design and measures. 
Heterogeneity will need to be within a recommended threshold, preferably where $P$ statistic <60\% (35). Statistical heterogeneity will also be assessed through forest plots, and the heterogeneity in effect sizes will be determined through Galbraith plots. If the studies are too heterogeneous, a narrative synthesis of the data will be presented and grouped by:

1. Individual-level

a. Mediation analyses

b. Moderation analyses

2. Multilevel
a. Mediation analyses
b. Moderation analyses

The Economic and Social Research Council Methods Programme guidelines (36) will be used to guide the synthesis and will allow identification of any sources of heterogeneity.

\section{Risk of bias}

Studies will be quality assessed using a modified version of the Newcastle-Ottawa Quality Assessment Scale (37). Assessments will include representativeness of participants, statistical methods implemented including identification of potential confounders, handling of missing data, attrition in longitudinal studies, how exposures and outcomes were measured, and any significant limitations of the study. If studies permit a meta-analysis, a funnel plot will be used to assess for publication bias.

\section{Reporting}

To allow for transparency and reproducibility of the findings, the methods and results of this systematic review will be reported according to the Preferred Reporting Items for Systematic Reviews and MetaAnalysis (PRISMA) guidelines (32).

\section{Timeline for systematic review}

It is anticipated that data extraction will start from $31^{\text {st }}$ October 2020 and a draft manuscript will be completed by January 2020.

\section{Discussion}

The quality and quantity of social relationships impact mortality, morbidity and mental health (7-8). Public Health England has acknowledged social isolation and social inequality as being related to poor health outcomes (38). Therefore, this systematic review will examine the link between social relationships and health inequalities. The indirect link between socioeconomic position and health via social relationships will be investigated with attention being paid to the mechanisms identified - whether social 
relationships mediate or moderate the association between socioeconomic position and health. The strengths and limitations of the evidence will be considered, and findings will be discussed in context with related reviews. The results of the review will summarise the existing evidence of the impact of social relationships on health inequalities and identify gaps in knowledge where further research is required. It is expected that the findings will be useful for the development of policies aiming to reduce social inequalities in health.

\section{Abbreviations}

WHO: World Health Organisation

PROSPERO: International prospective register of systematic reviews

PRISMA: Preferred Reporting Items for Systematic Reviews and Meta-Analyses

\section{Declarations}

\section{Acknowledgements}

The authors would like to thank Jacqui Smith for her help in developing the search strategy and selecting the databases. NK would also like to thank the Medical Research Council, her PhD funders.

\section{Authors' contributions}

All authors were involved in the conception and design of the protocol. NK drafted the manuscript, $\mathrm{AH}$ conceived the study, contributed to the study design and critically revised the manuscript. AM contributed to the study design and critically revised the paper. NK, AM, CMV and AH commented on drafts of the document, gave final approval for the manuscript to be published, they agree to be accountable and guarantors for all aspects of the work.

\section{Funding}

This work is supported by NK's PhD studentship from the UK Medical Research Council (grant number: MR/N013867/1).

\section{Competing interests}

None

Consent for publication 
Not applicable

\section{Ethics approval and consent to participate}

This study does not require ethical approval as it will draw on publicly available scientific literature.

Patient and public involvement

Patients or the public were not involved in the design, conduct, reporting or dissemination plans of this review protocol.

\section{Data statement}

Data sharing is not applicable to this article as no datasets were generated or analysed during the current study.

\section{References}

1. CSDH. Closing the gap in a generation: Health equity through action on the social determinants of health. Final Report of the Commission on Social Determinants of Health. 2008.

2. Solar, $\mathrm{O}$ and Irwin A. A Conceptual Framework for Action of the Social Determinants of Health: Social Determinants of Health Discuss Paper 2 (Policy and Practice). World Health Organisation 2010

3. Department of Health. Healthy Lives, Healthy People: Our strategy for public health in England. 2010.

4. Michael Marmot. Fair Society, Healthy Lives: The Marmot Review. Institute of Health Equity, London 2010

5. HM Government. Health and Social Care Act. 2012.

6. Public Health England. Reducing health inequalities: system, scale and sustainability About Public Health England. PHE Publ 2017;48.

7. Holt-Lunstad J, Smith TB, Layton JB. Social relationships and mortality risk: A meta-analytic review. PLoS Med. 2010;7(7).

8. Berkman LF, Krishna A. Social Network Epidemiology. In: Social Epidemiology. Berkman LF, Kawachi I, Glymour MM. Oxford University Press 2014.

9. Valtorta NK, Kanaan M, Gilbody S, Ronzi S, Hanratty B. Loneliness and social isolation as risk factors for coronary heart disease and stroke: Systematic review and meta-analysis of longitudinal observational studies. Heart 2016;102(13):1009-16.

10. Walker E, Ploubidis G, Fancourt D. Social engagement and loneliness are differentially associated with neuro-immune markers in older age: Time-varying associations from the English Longitudinal Study of Ageing. Brain Behav Immun. 2019; 82: 224-229 
11. Rouxel P, Tsakos G, Demakakos P, Zaninotto P, Chandola T, Watt RG. Is social capital a determinant of oral health among older adults? Findings from the English Longitudinal Study of Ageing. PLoS One 2015;10(5):1-17.

12. Stansfeld SA, Fuhrer R, Shipley MJ. Types of social support as predictors of psychiatric morbidity in a cohort of British civil servants (Whitehall II study). Psychol Med. 1998; 28(4):881-892.

13. Luanaigh CÓ, Lawlor BA. Loneliness and the health of older people. International Journal of Geriatric Psychiatry 2008; 23(12):1213-21

14. Cacioppo JT, Louise C. Hawkley. Perceived Social Isolation and Cognition. Trends Cogn Sci. 2009; 13(10):447-454.

15. Sampson EL, Bulpitt CJ, Fletcher AE. Survival of Community-dwelling older people: The effect of cognitive impairment and social engagement. J Am Geriatr Soc. 2009; 57(6):985-991.

16. Cassel J. The Contribution of the Social Environment to Host Resistance. Am J Epidemiol. 1976;104(2):1015-31.

17. House JS. Social Support and Social Structure. Sociol Forum 198;2(1):135-46.

18. Valtorta NK, Kanaan M, Gilbody S, Hanratty B. Loneliness, social isolation and social relationships: What are we measuring? A novel framework for classifying and comparing tools. BMJ Open 2016;6(4).

19. House, James S., Landis, Karl, R., Umberson D. Social Relationships and Health. Sci New Ser. 1988; 24:540-545.

20. Taylor SE, Seeman TE. Psychosocial resources and the SES-Health relationship. Ann N Y Acad Sci. 1999;896:210-25.

21. Vincens N, Emmelin M, Stafström M. Social capital, income inequality and the social gradient in selfrated health in Latin America: A fixed effects analysis. Soc Sci Med. 2018;196:115-22.

22. Tomazoni F, Vettore MV., Zanatta FB, Tuchtenhagen S, Moreira CH. The associations of socioeconomic status and social capital with gingival bleeding among schoolchildren. J Public Health Dent. 2017;77(1):21-9.

23. De Clercq B, Vyncke V, Hublet A, Elgar FJ, Ravens-Sieberer U, Currie C, et al. Social capital and social inequality in adolescents' health in 601 Flemish communities: A multilevel analysis. Soc Sci Med. 2012;74(2):202-10.

24. Aida J, Ando Y, Oosaka M, Niimi K. Contributions of social context to inequality in dental caries: A multilevel analysis of Japanese 3-year-old children. Community Dent Oral Epidemiol. 2008;36(2):149-56.

25. Aida J, Kondo K, Kondo N, Watt RG, Sheiham A, Tsakos G, et al. Income inequality, social capital and self-rated health and dental status in older Japanese. Soc Sci Med. 2011;73(10):1561-8.

26. Craveiro $D$. The role of personal social networks on health inequalities across European regions. Heal Place 2017;45:24-31. 
27. Carlson ED, Chamberlain RM. Social Capital, Health and Health Disparities. J Nurs Scholarsh. 2003;35(4):325-31.

28. Uphoff EP, Pickett KE, Cabieses B, Small N, Wright J. A systematic review of the relationships between social capital and socioeconomic inequalities in health: A contribution to understanding the psychosocial pathway of health inequalities. Int J Equity Health. 2013;12(1):54.

29. Vyncke V, De Clercq B, Stevens V, Currie Costongs C, Barbareschi G, Jonsson SH, et al. Does neighbourhood social capital aid in levelling the social gradient in the health and well-being of children and adolescents? A literature review. BMC Public Health 2013;13:65.

30. Moher D, Shamseer L, Clarke M, Ghersi D, Liberati A, Petticrew M, et al. Preferred reporting items for systematic review and meta-analysis protocols (prisma-p) 2015: Elaboration and explanation. BMJ 2015;

31. Holt-Lunstad J, Robles TF, Sbarra DA. Advancing social connection as a public health priority in the United States. Am Psychol. 2017;72(6):517-30.

32. Moher D, Liberati A, Tetzlaff J AD. PRISMA 2009 Flow Diagram. The PRISMA statement. 2009.

33. Foeckler P, Henning V, Reichelt J. Mendeley. 2008.

34. Ouzzani M, Hammady H, Fedorowicz Z, Elmagarmid A. Rayyan-a web and mobile app for systematic reviews. Syst Rev. 2016;5(1):1-10.

35. Deeks JJ, Higgins J, Green S. Chapter 9: Analysing data and undertaking meta-analyses. In: Higgins JPT, Green S (editors). In: Cochrane Handbook for Systematic Reviews of Interventions. 2011.

36. Jennie Popay, Helen Roberts, Amanda Sowden, Mark Petticrew, Lisa Arai, Mark Rodgers, et al. Guidance on the Conduct of Narrative Synthesis in Systematic Reviews. ESRC Methods Programme. 2006.

37. Wells G, Shea B, O'Connell D, Peterson J. The Newcastle-Ottawa Scale (NOS) for assessing the quality of nonrandomised studies in meta-analyses. Ottawa, ON: Ottawa Hospital Research Institute. 2000.

38. Durcan D, Bell R. Reducing social isolation across the lifecourse. 2015.

\section{Supplementary Files}

This is a list of supplementary files associated with this preprint. Click to download.

- AdditionalFile1BMCResearchChecklist.pdf

- AdditionalFile3BMCSearchStrategyToolsTechniques.pdf

- AdditionalFile2BMCSearchTerms.pdf 